\title{
Erwinia carotovora Evf antagonizes the elimination of bacteria in the gut of Drosophila larvae
}

\author{
Carlos Acosta Muniz, ${ }^{1}$ Danielle Jaillard, ${ }^{2}$ Bruno \\ Lemaitre $^{1 *}$ and Frédéric Boccard ${ }^{1 *}$ \\ ${ }^{1}$ Centre de Génétique Moléculaire du CNRS, Bâtiment \\ 26, 1 Avenue de la Terrasse, 91198 Gif-sur-Yvette, \\ France. \\ ${ }^{2}$ Unité Mixte de Recherche 8080 du CNRS, Bât. \\ 440 bis, Université Paris XI, 91405 Orsay, France.
}

\section{Summary}

Erwinia Virulence Factor (Evf) has been identified in Erwinia carotovora carotovora 15 (Ecc15) as a virulence factor that promotes colonization of the Drosophila larval gut and provokes the triggering of a systemic immune response. Here we have analysed how Evf promotes persistence and colonization of bacteria inside the larval gut. Erwinia evf mutants do not persist in immune-deficient Drosophila, indicating that Evf does not act by counteracting immunity. The results indicated that Evf is not a toxin because various Gram-negative bacteria expressing evf can persist without affecting viability of Drosophila larvae. Evf did not appear to be a factor antagonizing a host-specific reaction because in vitro assays failed to reveal detoxifying enzymatic activities against various compounds thought to contribute to the hostile environment of the gut. These findings were corroborated by the observation that Evf is not required for survival in midgut organ cultures. By contrast, bacteria expressing evf allow persistence in trans of bacteria lacking evf indicating that Evf promotes the accumulation of Gram-negative bacteria in the anterior midgut by affecting gut physiology.

\section{Introduction}

In recent years, a number of genetically amenable organisms have been used as models to study host/pathogen interactions. The fruit fly Drosophila has been very useful in characterizing signalling pathways and mechanisms used by the host to prevent and combat microbial

Received 16 April, 2006; revised 8 June, 2006; accepted 13 June, 2006. *For correspondence. E-mail lemaitre@cgm.cnrs-gif.fr; Tel. (+33) 1698232 27; Fax (+33) 169824386 or email boccard@ cgm.cnrs-gif.fr; Tel. (+33) 1698232 11; Fax (+33) 169823150.

(C) 2006 The Authors

Journal compilation @ 2006 Blackwell Publishing Ltd infection. The Drosophila immune response consists of both cellular and humoral responses. Expression of immune effectors is mainly under the control of two signalling pathways designated Toll and Imd (Hultmark, 2003). The Toll pathway is predominantly activated by Gram-positive bacteria and fungi, and induces the synthesis of several peptides including the antifungal peptide drosomycin. On the other hand, the Imd pathway is activated predominantly by Gram-negative bacteria and induces the expression of different antimicrobial peptides encoding genes (e.g. diptericin). In addition, the Imd pathway controls the local expression of antimicrobial peptides in epithelia such as gut or trachea. Up to recently, most studies have involved the direct injection of microbes into the insect body cavity. In the last few years, a second approach called natural infection has been developed to mimic infections as they probably occur in nature (Basset et al., 2000). This method consists of feeding Drosophila larvae or adults with food containing a high bacterial titre. Isolation of bacteria that elicit an immune response after ingestion might reveal strategies that are used by microbes to persist in their host, especially the initial steps of infection (Vodovar et al., 2004).

Upon ingestion, most bacterial strains appear to be non-infectious, i.e. they do not persist in the fly and/or do not induce an immune response. Only a few microbes have been described as being able to trigger the immune response or to be pathogenic. These include Serratia marcescens (Flyg et al., 1980), a qscR mutant of Pseudomonas aeruginosa (Chugani et al., 2001), and Pseudomonas entomophila (Vodovar et al., 2005). In previous studies, we have shown that several Erwinia species were able to elicit the immune response (Basset et al., 2000). Among these, Erwinia carotovora carotovora 15 (Ecc15) is able to persist in the gut of larvae and induces both a local and systemic immune response while not killing the larvae. By using a genetic screen, we have identified two genes that were required by Ecc15 to infect Drosophila (Basset et al., 2003). The first gene encodes a global regulator, Hor, and seemed to exert its effect by regulating the second identified gene, evf. Erwinia Virulence Factor (Evf) may play a role in gut persistence as its transfer into different enterobacteria makes them infectious for Drosophila. No homologous genes were found in other organisms and no domains with predicted activity or signature could be discerned in Evf. 
A
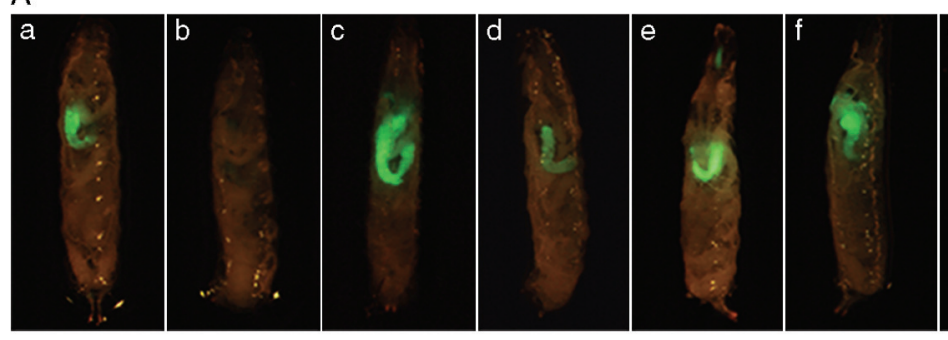

g

B

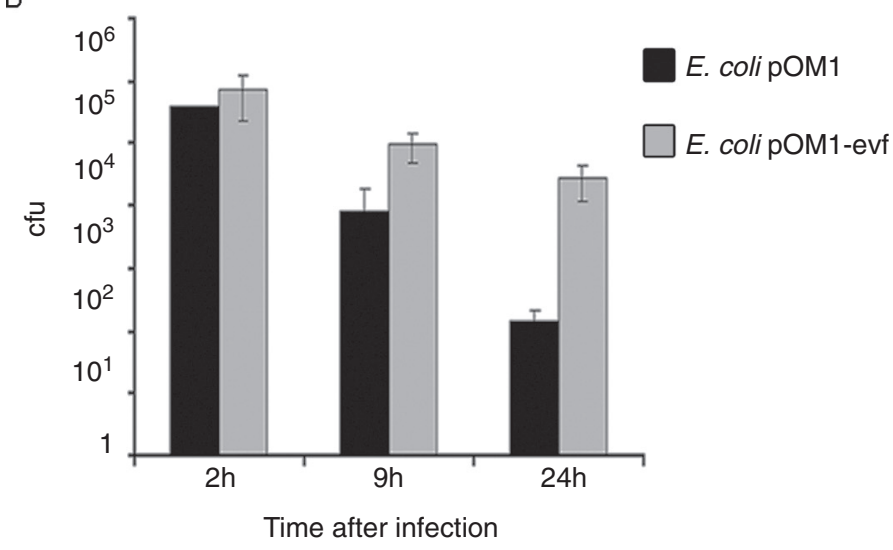

Fig. 1. Effect of Evf on the persistence of Gram-negative bacteria in the gut.

A. Wild-type (OrR) larvae were naturally infected with various bacteria expressing a GFP reporter gene. (a) Ecc15 carrying pOM1-GFP, (b) Ecc15 evf mutant carrying pOM1-GFP, (c) Ecc15 evf mutant carrying pOM1-evf-GFP, (d) S. typhimurium pOM1-evf-GFP, (e) E. coli pOM1-evf-GFP, (f) $P$. aeruginosa PAO1 carrying $\mathrm{pX}$-evf-GFP and $(g) P$. aeruginosa carrying $\mathrm{pX}$-gfp. Pictures were taken $6 \mathrm{~h}$ after infection. B. Bacterial persistence was measured in wt larvae. Bacterial counts were obtained by plating, on LB medium containing spectinomycin $\left(100 \mu \mathrm{g} \mathrm{ml}^{-1}\right)$, the larval homogenates of five surface-sterilized larvae that were naturally infected with $E$. coli carrying pOM1 and E. coli carrying pOM1-evf. The number of colony forming units (cfu) per larva obtained at each point after infection represents the mean of three independent measurements.
Here we describe a number of experiments performed to understand how Evf allows bacteria to persist in the Drosophila larval gut. We show that Evf activity relies on the presence of Evf in the cytoplasm of Gram-negative bacteria. Our results indicate that persistence of bacteria in the gut does not involve a detoxifying activity directed against the host immune system but rather leads to a modification of insect gut physiology that is under normal circumstances, responsible for the eradication of ingested bacteria.

\section{Results}

\section{Evf allows persistence of Gram-negative bacteria in the gut of Drosophila}

In a previous study, we have shown that transfer of evf to different enterobacteria, i.e. Escherichia coli, Salmonella typhimurium and Serratia marcescens, transformed these bacteria into infectious microbes that induced a strong antibacterial response upon ingestion (Basset et al., 2003). To further characterize the interaction of these bacteria with the fly, Drosophila larvae were fed with bacteria expressing both evf and the gene encoding green fluorescent protein (GFP), gfp. Whereas overexpression of evf in Ecc15 induced a strong lethality in Drosophila $12 \mathrm{~h}$ after feeding, no lethality was apparent with the three other enterobacteria. All enterobacteria were present in the gut after $6 \mathrm{~h}$ and no fluorescence was apparent in other tissues of the fly (Fig. 1A). The persistence of bacteria in the gut was more precisely estimated by plating gut extracts. In the case of wild-type (wt) E. coli cells, the titre of bacteria decreased from $10^{6}$ after $1 \mathrm{~h}$ to $10^{4}$ after $9 \mathrm{~h}$ and $10^{2}$ after $24 \mathrm{~h}$. By contrast, the titre of E. coli cells expressing evf remained high, between $10^{4}$ and $10^{5}$ after 9 or $24 \mathrm{~h}$ (Fig. 1B). This level is similar to that obtained with Ecc15 overexpressing evf (Basset et al., 2003).

The same experiment was performed with various Pseudomonas species, Gram-negative bacteria that are more distantly related to enterobacteria. In the absence of evf, Pseudomonas species such as $P$. aeruginosa PAO1 or Pseudomonas putida KT2440 did not persist and did not induce an immune response as previously described (Vodovar et al., 2005). P. aeruginosa PAO1 (Fig. 1A) and $P$. putida KT2440 (data not shown) expressing evf persisted in the gut, induced a strong antibacterial response and provoked lethality after $12 \mathrm{~h}$ (data not shown).

The ability of Evf to confer infectivity was tested for different Gram-positive bacteria. evf was placed under the control of the promoter $\mathrm{P}_{\text {SPAC }}$ and inserted in the plasmid pDG148 expressing gfp (see Experimental procedures). This recombinant plasmid was transformed in Bacillus megaterium, Bacillus subtilis and Streptococcus faecalis. In all cases, whereas fluorescence was apparent in the gut upon ingestion, no fluorescence remained visible after $6 \mathrm{~h}$ and no Toll-dependent or Imd-dependent immune responses were detected (data not shown). 
Whereas the bacterial entomopathogen $P$. entomophila is able to infect Drosophila but also additional species belonging to different insect orders (Vodovar et al., 2005), the host range of other entomopathogens such as Bacillus thuringiensis is more restricted (de Maagd et al., 2001). To determine the host range against which Evf can confer infectious properties, larvae of various Drosophila species ( $D$. virilis, D. busckii, D. bifascata, D. simulans) and of two lepidopteran species (Bombyx mori and Galleria mellonella) were infected by Ecc15 carrying a pOM1-evf plasmid. Ecc15 expressing evf were able to persist in all of the tested Drosophila species and lethality was apparent after $12 \mathrm{~h}$. By contrast, these bacteria were not able to persist in either Bombyx or Galleria (data not shown). Collectively, our data indicate that evf is a specific Gram-negative virulence factor that promotes colonization to a restricted niche, the gut of Drosophila larvae.

\section{Regulation of synthesis and localization of Evf}

We have previously shown that evf expression requires Hor (Basset et al., 2003), a general regulator of virulence in various enterobacteria (Thomson et al., 1997). However, no canonical promoter sequence could be identified in the upstream region of evf. In order to determine the extent of sequences required for evf expression, we fused different fragments of various length from that region (0, 150 and 300 nucleotides) to the lacZ gene (Fig. 2A). The activity of these constructs was tested in different genetic backgrounds, i.e. in wt Ecc15, in hor mutants and in an E. coli derivative with lac $Z$ deleted. In the control with no fragment inserted upstream of $l a c Z$, no $\beta$-gal activity was detected in Ecc15. While the construct carrying the region extending up to $150 \mathrm{bp}$ gave rise to a low level of $\beta$-gal activity, constructs carrying the region extending up to $300 \mathrm{bp}$ upstream of evf promoted the highest amount of $\beta$-gal activity. This activity was lost in a hor mutant indicating that the 300 -bp-long region upstream of the evf coding sequence contains the information for promoter activity and sites required for Hor regulation. In E. coli cells that do not possess the hor gene, the level of $\beta$-gal activity was similar to that obtained with the hor mutant revealing a low Horindependent promoter activity. As pOM1 derivatives are present at about 10 copies per cell, these results indicate that in Ecc15, the wt level of Evf corresponds to a level of about 60 units $(u)$ of $\beta$-gal in the exponential growth phase (Fig. 2B) and reached $120 \mathrm{u}$ in the stationary growth phase (data not shown). By extrapolation from results obtained in E. coli (Deng et al., 2004), these data indicate a steady state abundance of about three copies of RNA per DNA molecule in wt Ecc15. From the level obtained in stationary phase in the hor mutant carrying
A
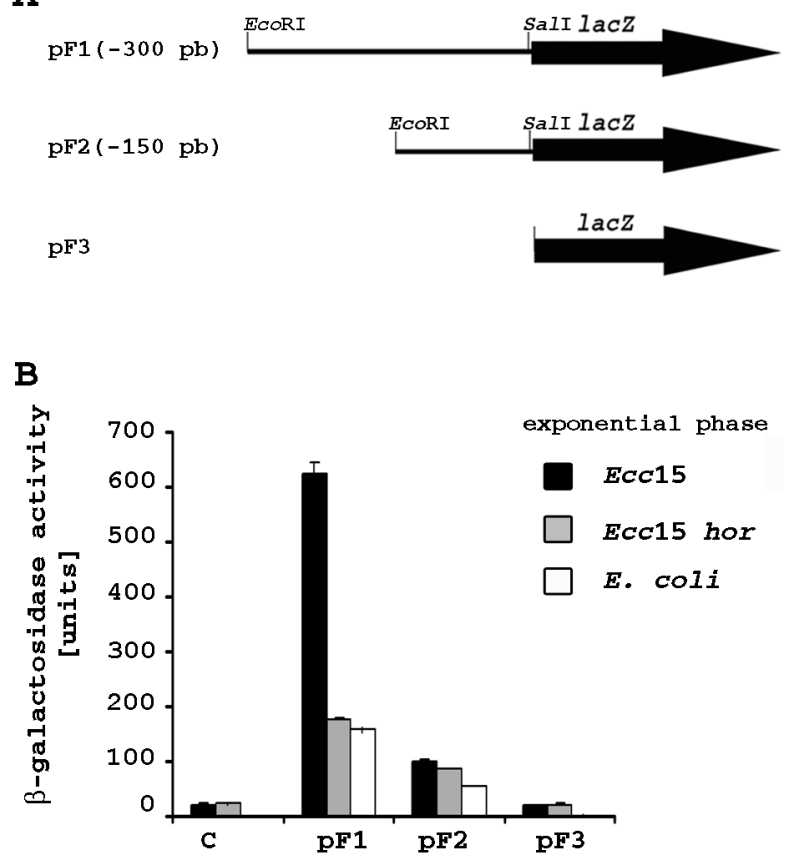

Fig. 2. Level of expression of evf required to infect Drosophila. A. Analysis of the evf promoter region. Various lengths of the upstream region of evf were amplified by PCR and cloned upstream of a lac $Z$ reporter gene. The numbers given in brackets denote the size of the evf upstream region in the different constructs $\mathrm{pF} 1, \mathrm{pF} 2$ and $\mathrm{pF} 3$.

B. Analysis of expression mediated by different regions of the evf-lacZ reporter gene in Ecc15, Ecc15 hor mutant and in an E. coli derivative deleted for lacZ. The $\beta$-galactosidase activities represent the mean \pm SD of three independent experiments. Control activity (C) corresponds to the background level of the strains.

the construct with the 300-bp-long region upstream of the evf coding sequence ( $400 \mathrm{u}$, data not shown), we can deduce that a level of $40 \mathrm{u}$ is synthesized from the chromosomal copy of evf in the hor mutant. As this mutant is not infectious, the minimal expression level required for infection should be greater than $40 \mathrm{u}$ and less or equal to $120 \mathrm{u}$ (the level calculated from a single copy in Ecc15 in stationary phase).

Evf activity could not be predicted from its primary sequence. A clue to the Evf action leading to bacterial persistence was the identification of its subcellular localization in the cell. Interestingly, Evf contains a putative transmembrane domain (residues 128-148) predicted to target the $\mathrm{C}$-terminal domain of the protein to the periplasm and the $\mathrm{N}$-terminal domain to the cytoplasm. To verify the predicted localization, we constructed two classes of gene fusions (Fig. 3); one class fuses various parts of evf to $l a c Z$, the other one various parts of evf to phoA encoding phosphatase alkaline. $\beta$-Gal activity should be detected if Evf is present in the cytoplasm whereas no activity should be found if Evf is associated with the membrane or targeted to the periplasm. By 
A

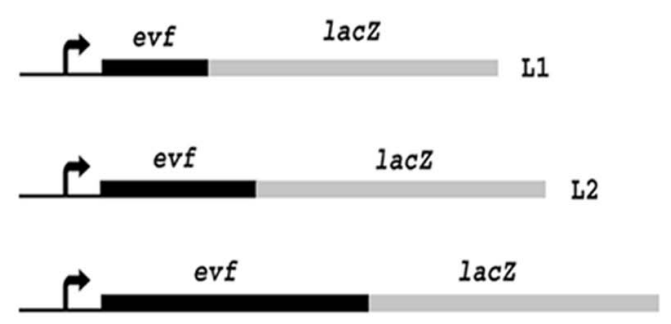

C

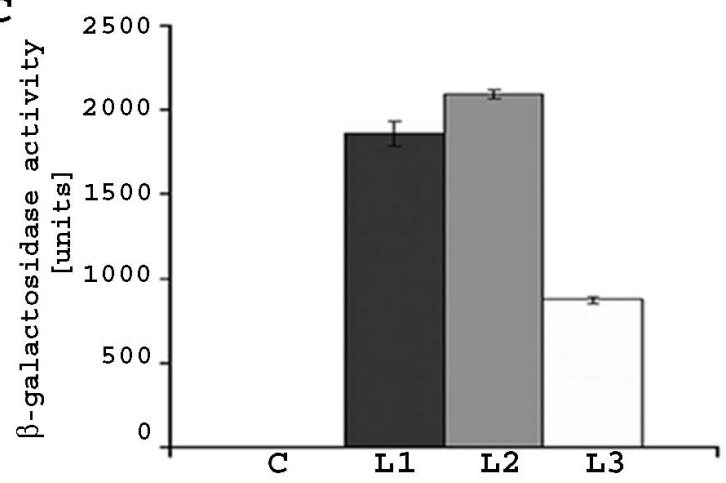

B

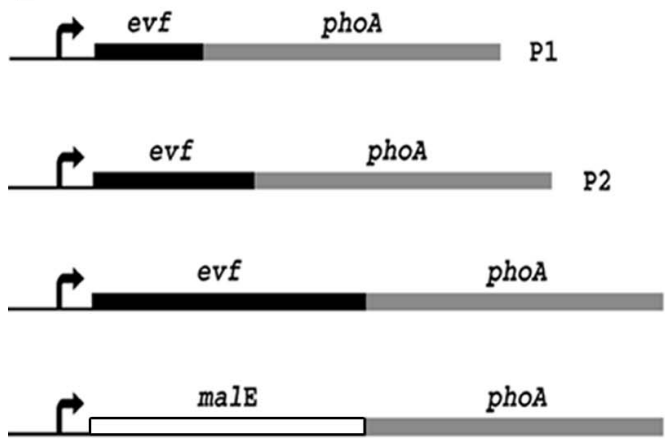

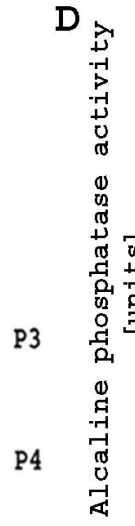

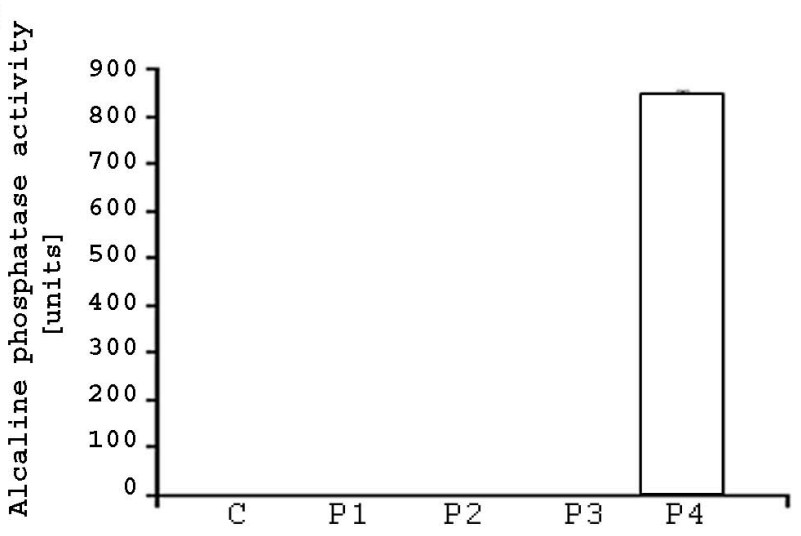

Fig. 3. Cellular localization of Evf.

$\mathrm{A}$ and $\mathrm{B}$. Translational fusions of evf to $\operatorname{lac} Z(\mathrm{~L})$ and $p h o A(\mathrm{P})$. L1 and $\mathrm{P} 1$ fusions carry the N-terminal region of Evf predicted to have a cytoplasmic localization. The L2 and P2 fusions code for the $\mathrm{N}$-terminal region together with the predicted transmembrane domain of Evf. The L3 and P3 fusions carry the full-length evf. The P4 fusion is a control that contains the signal peptide of MalE responsible for the export of MalE into the periplasm. The vectors pMC1403 and pPHO7 were used for the evf-lacZ and evf-phoA fusions respectively.

$C$ and D. $\beta$-Galactosidase and phosphatase alkaline activities of different fusions of evf indicate a cytoplasmic localization of Evf. The $\beta$-galactosidase and phosphatase alkaline activities represent the mean \pm SD of three independent measurements. Control activity (C) corresponds to the background level of the strains carrying the plasmid control.

contrast, PhoA activity would indicate a periplasmic localization of the fusion (Manoil, 2000). evf-lacZ fusions all gave rise to $\beta$-gal activity regardless the site of fusion in evf (Fig. 3C). Conversely, no PhoA activity was detected with the different fusions. A control malE-phoA fusion containing the signal for periplasm localization of MalE gave rise to PhoA activity (Fig. 3D). Altogether, the genetic approach predicted that Evf is synthesized in the cytoplasm and no association with the periplasm was found. This result was further confirmed by Western blot analyses using protein extracts from different subcellular compartments and antibodies directed against Evf (data not shown).

\section{Is Evf sufficient to confer infectivity to Gram-negative bacteria?}

The ability of evf to confer infectivity to different, normally non-persistent, Gram-negative bacteria such as
Pseudomonas or Erwinia species suggested at least two alternative modes of action. First we could imagine that Evf indirectly protects bacteria in the gut of larvae by modifying some metabolic pathways or by activating a transcriptional network that renders the bacterial cells refractory to elimination by the host immune system or other harmful molecules in the gut. Alternatively Evf could exert its action autonomously by modifying directly a host effector participating in bacterial clearance. If the first hypothesis is true, we would expect that several E. coli genes are involved in this metabolic pathway and we should be able to isolate $E$. coli mutants expressing evf that are unable to infect Drosophila larvae. To this end, we constructed an E. coli strain carrying a single evf-lac $Z$ operon cloned downstream of the $P_{R}$ promoter integrated in the chromosome (see Experimental procedures). The rationale behind the insertion of lac $Z$ downstream of evf was to directly identify mutants resulting from Tn10 transposition in evf. Three thousand E. coli 
Table 1. Sensitivity of Ecc15 derivatives to various reactive oxygen and nitrogen intermediates.

\begin{tabular}{|c|c|c|c|c|}
\hline \multirow[b]{2}{*}{ Compound } & \multirow[b]{2}{*}{ Concentration } & \multicolumn{3}{|c|}{ Diameter $(\mathrm{mm})$ of growth inhibition zone $(\text { mean } \pm \mathrm{SD})^{\mathrm{a}}$} \\
\hline & & Ecc15 pOM1 & Ecc15 evf pOM1 & Ecc15 evf pOM1-evf \\
\hline Paraquat & $2 \%$ & $18.0 \pm 0.5$ & $18.0 \pm 0.5$ & $18.5 \pm 1.0$ \\
\hline $\mathrm{H}_{2} \mathrm{O}_{2}$ & $250 \mathrm{mM}$ & $21.0 \pm 0.5$ & $21.0 \pm 0.5$ & $21.0 \pm 0.5$ \\
\hline $\mathrm{HOCl}$ & $5 \%$ & $25.0 \pm 0.1$ & $25.0 \pm 0.5$ & $25.0 \pm 0.5$ \\
\hline GSNO & $1 \mathrm{M}$ & $13.0 \pm 0.2$ & $12.5 \pm 1.0$ & $13.0 \pm 0.3$ \\
\hline SIN-1 & $1 \mathrm{M}$ & $9.0 \pm 0.1$ & $9.0 \pm 0.1$ & $9.0 \pm 0.1$ \\
\hline SNAP & $500 \mathrm{mM}$ & $10.5 \pm 0.2$ & $10.0 \pm 0.5$ & $10.5 \pm 0.2$ \\
\hline Spermin/NONOate & $1 \mathrm{M}$ & $15.0 \pm 0.3$ & $15.0 \pm 0.6$ & $17.0 \pm 0.5$ \\
\hline DETA/NONOate & $1 \mathrm{M}$ & $20.0 \pm 0.6$ & $20.0 \pm 0.6$ & $20.0 \pm 0.3$ \\
\hline
\end{tabular}

a. Growth inhibition zones around $6 \mathrm{~mm}$ diameter disks soaked with $10 \mu \mathrm{l}$ of the different solutions were measured after overnight incubation. The values are the averages of three measurements.

variants were generated using pNKBOR, a mini-Tn10 derivative (see Experimental procedures). These 3000 variants were individually tested in Drosophila larvae for the inability to induce a Diptericin-gfp fusion, a read-out that correlates with the capacity to infect the host. Among the 3000 variants, two non-infectious variants were identified, which were deficient for $\beta$-gal activity, i.e. NKBOR affected directly evf. All remaining 2998 lac+ variants were infectious. We concluded from this experiment that genes belonging to a pathway putatively affected by Evf could not be identified using this approach. To directly identify genes whose expression might be modified by the presence of Evf, we compared the transcriptome of E. coli cells expressing evf and that of wt $E$. coli cells. No specific and reproducible changes of gene expression were detected indicating that Evf does not significantly affect the bacterial transcriptome (data not shown).

We therefore considered the possibility that evf could exert its activity directly, for example by detoxifying or neutralizing harmful molecules present in the Drosophila gut. A set of tests was developed to determine whether Evf could confer resistance or allow adaptation to hostile conditions, more specifically to reactive oxygen species (ROS), acid or alkaline stress, ethanol stress, osmotic stress, or resistance to trypsin or lysozyme treatment. Ecc15 strains behaved similarly to all reactive nitrogen and oxygen intermediates conditions independently of the presence or of the absence of evf expression (Table 1). It is interesting to note that Ecc15 was more susceptible to paraquat, and as susceptible to $\mathrm{H}_{2} \mathrm{O}_{2}$ or $\mathrm{HOCl}$ as $P$. putida that does not persist in the larval gut. These results suggest that although oxidative stress plays an important role in clearance of bacteria in the gut of adult insects $\mathrm{Ha}$ et al., 2005a,b), Evf does not act in larvae by counteracting this eradication process. Similarly, no significant differences were detected for the other types of stress (data not shown). Altogether, our results support the idea that Evf is a direct effector promoting persistence, while this effect cannot be explained simply by a protection against a chemical stress.

\section{Nature of the activity of Evf}

Ecc15 do not persist in wt Drosophila larvae and the number of bacteria after $24 \mathrm{~h}$ is reduced by a factor $10^{3}$ (Basset et al., 2000). In contrast, the number of bacteria was reduced only 10-fold in imd-deficient Drosophila lines unable to induce an antibacterial response, remaining high at a level of $10^{5}$ and $10^{6}$ after $24 \mathrm{~h}$ (Basset et al., 2000 and Fig. 4A). This demonstrates a role for Imd pathway-dependant immune responses in the control of Ecc15. To determine whether Evf activity antagonizes early events of the host antibacterial response, we monitored the persistence of Ecc15 evf mutants in Imddeficient Drosophila larvae (Fig. 4A). In the absence of a functional Imd pathway, evf mutants were not able to persist. This indicates that Evf does not promote persistence by directly counteracting the Imd-dependant immune response. Of note, E. coli cells lacking evf do not persist in Relish flies after oral infection (data not shown). Altogether, this indicates that Evf does not target the larval antimicrobial peptide defence.

It is generally assumed that the Drosophila gut constitutes a hostile environment unfavourable for bacterial persistence. To determine whether Evf affects persistence in this environment, we isolated the gut immediately after ingestion of bacteria synthesizing GFP, maintained them in a physiological buffer and followed the persistence of fluorescent bacteria up to $24 \mathrm{~h}$. Strikingly, under these conditions, evf mutants persisted as well as bacteria expressing evf (Fig. 4B). These results therefore indicate that elimination of bacteria requires the maintenance of the gut in the body of the larvae and may involve complex physiological properties such as peristaltic flushing.

Antagonizing the peristaltic movements or other mechanical processes eliminating ingested microbes 
(A)

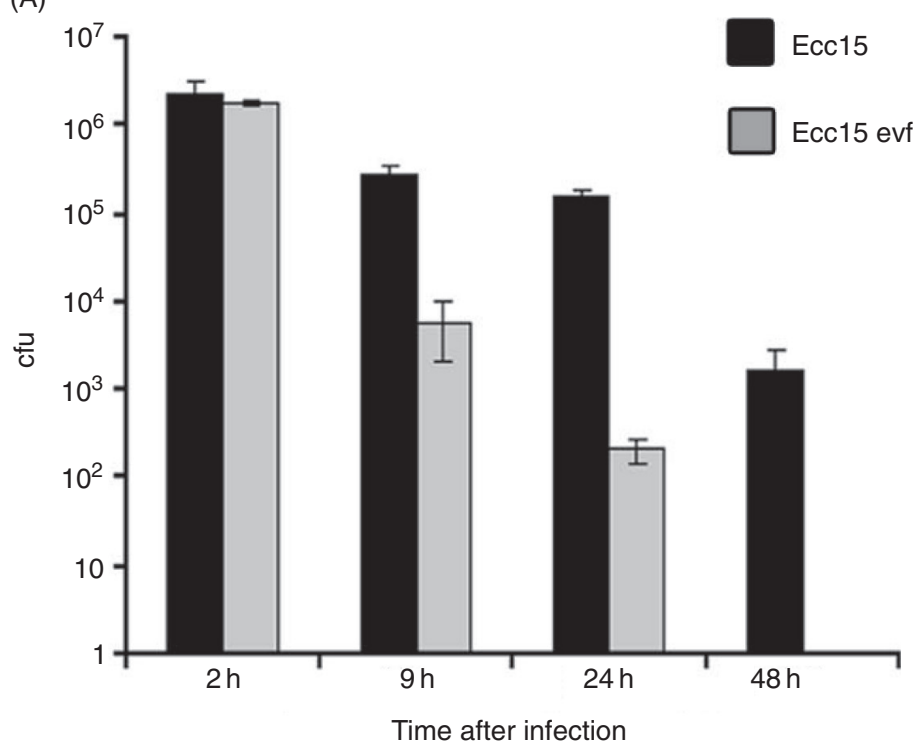

(B)

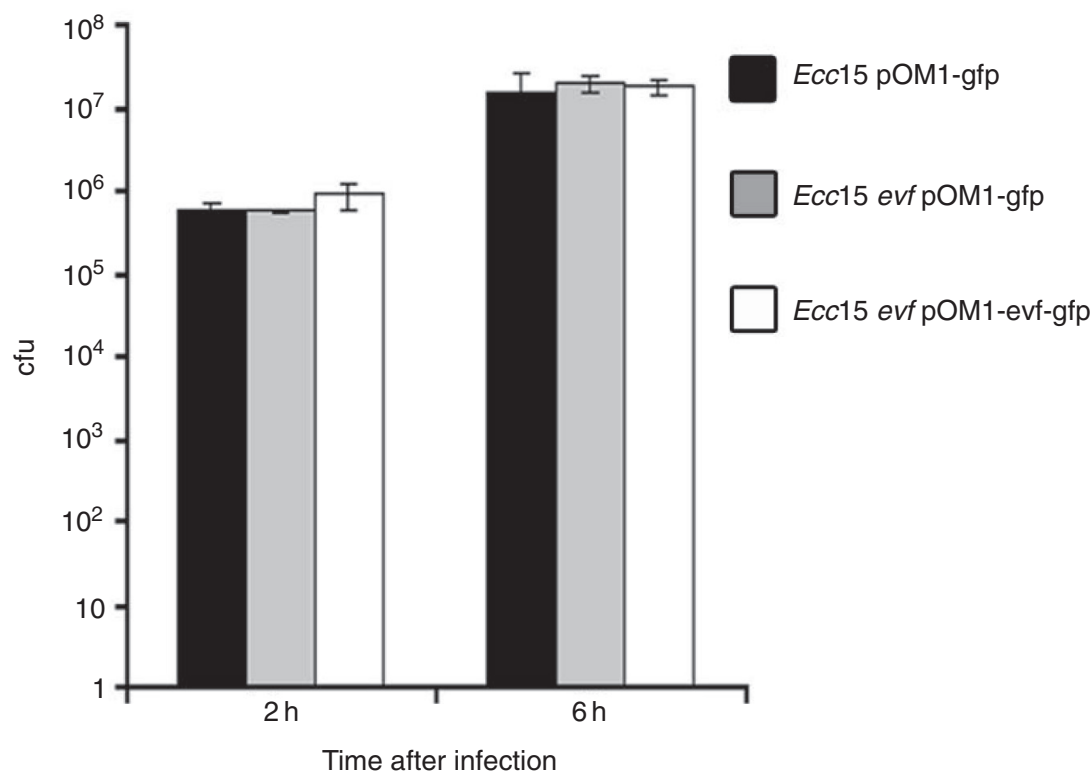

Fig. 4. Persistence of evf mutant bacteria in Relish larvae and isolated gut preparations.

A. Persistence of Ecc15 evf mutant in Relish larvae. Bacterial persistence was measured by plating appropriate dilutions of homogenates of five surface-sterilized larvae that were naturally infected with rifampicin-resistant strains of $E c c 15$ and Ecc15 evf mutant. Bacterial counts were obtained by plating the larval homogenates on LB agar containing $100 \mu \mathrm{g} \mathrm{ml}^{-1}$ of rifampicin. The number of colony forming units (cfu) per larva obtained at each point after infection represents the mean of three independent measurements.

B. Persistence of Ecc15 evf mutants in isolated gut. Bacterial persistence was measured in isolated guts of wt larvae by plating appropriate dilutions of homogenates of five isolated guts of larvae that were naturally infected with Ecc15 pOM1-gfp, Ecc15 evf pOM1-gfp and Ecc15 evf pOM1-evf-gfp. Larvae were orally infected by bacteria, and, at $2 \mathrm{~h}$ post infection, the gut of larvae were dissected and incubated in Schneider medium. Bacterial counts were obtained by plating the gut homogenates collected at 2 and $6 \mathrm{~h}$ post infection on LB agar containing $100 \mu \mathrm{g} \mathrm{ml}^{-1}$ of spectinomycin. The number of cfu per gut obtained at each point after infection represents the mean of three independent measurements. should allow the persistence of other bacteria in trans. To test this hypothesis, we performed a set of oral infections using bacterial mixtures containing either fluorescent Ecc15 alone, fluorescent Ecc15 evf mutant alone, or Ecc15 together with fluorescent Ecc15 evf mutant bacteria (Fig. 5). As expected, fluorescence was still detected $6 \mathrm{~h}$ after ingestion of Ecc15 whereas no fluorescence was detected at the same time with fluorescent evf- bacteria (Fig. 5A). Remarkably, $6 \mathrm{~h}$ after ingestion, fluorescence was evident when fluorescent evf mutants were coinfected with Ecc15. These results indicate that
Evf antagonizes directly or indirectly the processes responsible for bacterial eradication. Plating bacteria isolated from infected gut allowed direct counting of bacteria and confirmed observations of GFP fluorescence, because the titre of Ecc15 evf mutant bacteria was reduced by a factor $10^{3} 6 \mathrm{~h}$ post infection (Fig. 5B), while this level remained high in the presence of evfexpressing bacteria. Similar effects of Evf on persistence in trans were obtained when Erwinia strains were substituted by E. coli strains (data not shown). Altogether, our results indicate that evf-expressing bacteria 
A

$2 \mathrm{~h}$
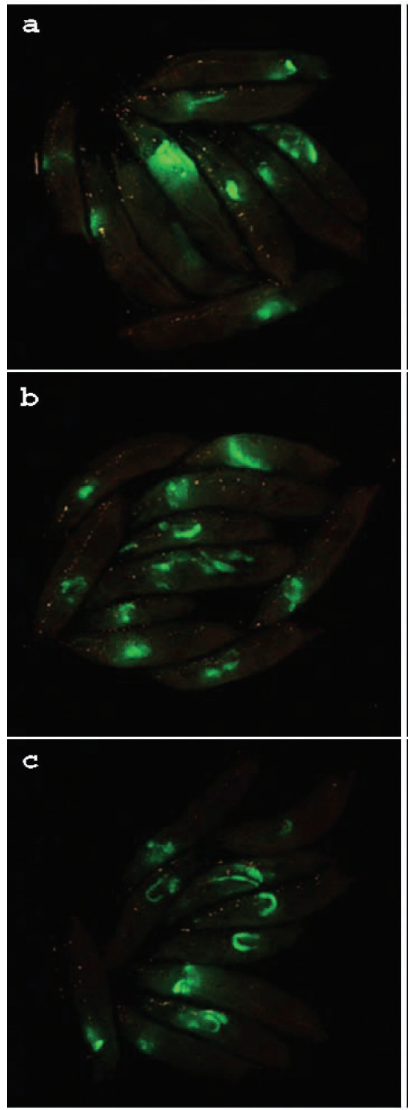

B

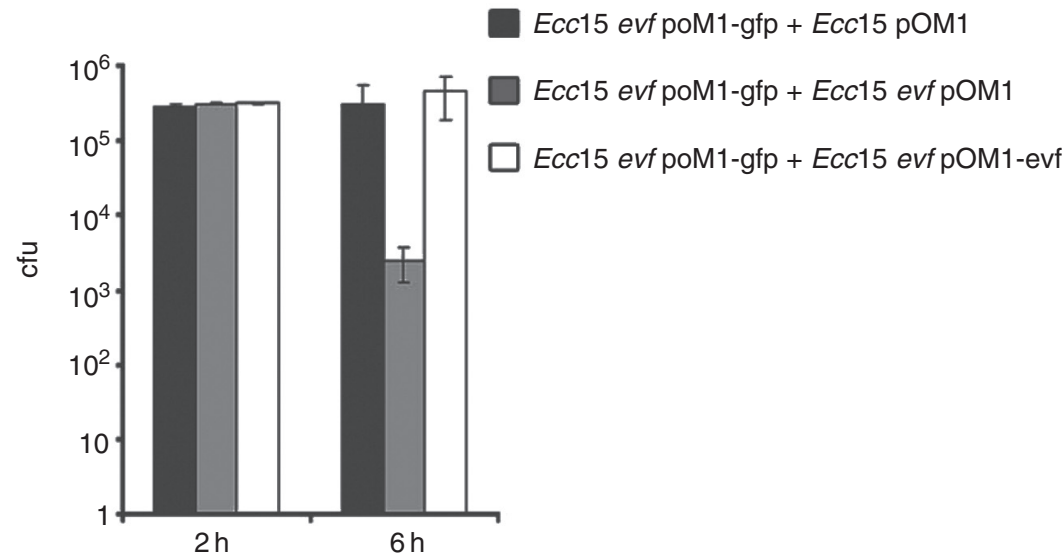

$6 \mathrm{~h}$

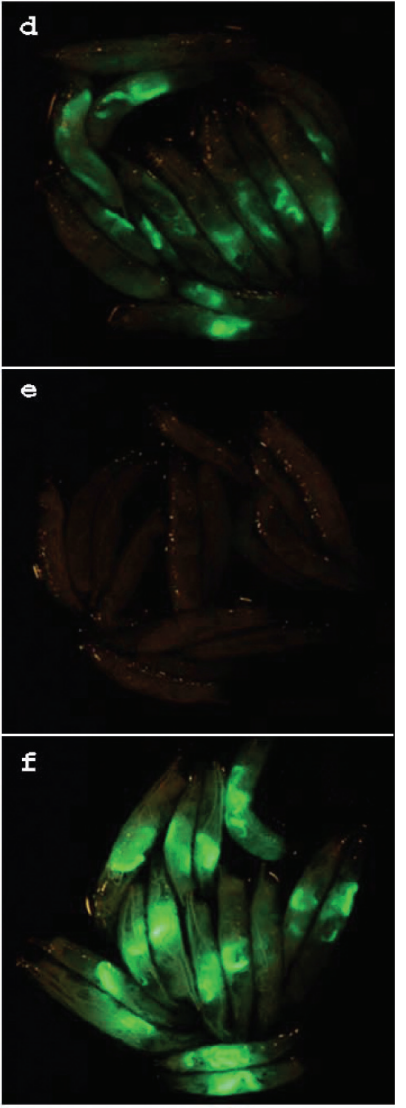

Fig. 5. Persistence of non-infectious bacteria by evf-expressing bacteria.

A. Persistence of evf mutants coinfected with bacteria expressing evf in wt larvae. Larvae were photographed at $2 \mathrm{~h}(\mathrm{a}, \mathrm{b}, \mathrm{c})$ and $6 \mathrm{~h}(\mathrm{~d}$, e, f) post infection. ( $a$ and d) GFP expressing bacteria in larvae infected with a mixture of Ecc15 pOM1 + Ecc15 evf pOM1-GFP (b and e) larvae infected with a mixture of Ecc15 evf and Ecc15 evf carrying pOM1-gfp, and (c and f) larvae infected with a mix of Ecc15 evf pOM1-evf and Ecc15 evf pOM1-gfp.

B. Bacterial counts were obtained by plating the larval homogenates of five

surface-sterilized larvae that were naturally infected with the mixtures of bacteria on LB medium containing spectinomycin

$\left(100 \mu \mathrm{g} \mathrm{ml}^{-1}\right)$. The number of colony forming units (cfu) per larva obtained at each point after infection represents the mean of three independent measurements.

Time after infection

promoted efficient persistence of non-infectious bacteria in trans.

Overexpression of evf promotes bacterial accumulation in the gut and induces lethality

Ecc15 bacteria carrying evf under the control of the promoter Pro3 in a pSC101 plasmid derivative (Espeli et al.,
2001) express evf about 50 times more efficiently than wt Ecc15. Under these conditions, bacteria were able to persist for a longer period and at a higher level than wt Ecc15 (more than 10 times, Basset et al., 2003). By using a derivative expressing gfp, we were able to visualize bacterial accumulation predominantly in the gut (Fig. 1 and Basset et al., 2003). To observe in greater detail the consequences of accumulating Ecc15 derivatives in the 
gut, we performed histological analyses at different time points following ingestion of bacteria. Electron and optic micrographs of transversal sections of larval midguts revealed a high accumulation of bacteria in the gut lumen delimited by the peritrophic matrix (Fig. 6C). At 2 h 30 min after infection, the mucus that protects the digestive epithelium was absent in larvae infected with Ecc15 pOM1evf (Fig. 6C and D) compared with the non-infected control (Fig. 6A and B). At time $6 \mathrm{~h}$, the gut lumen was filled with bacteria that appeared in regular arrangement, the peritrophic matrix lining the epithelial cells was intact, and high quantities of cellular material seemed to be present in the space between the epithelial cells and the peritrophic matrix (Fig. 6F-H).

The high numbers of bacteria in the gut revealed the ability of Evf to promote bacterial persistence at least in this part of the animal. To determine the effect of Evf on the outcome of an infection in other tissues, bacteria overexpressing evf were injected directly in the haemocoele of adult flies and larvae, which did not lead to apparent lethality (data not shown). These results therefore indicated that evf-conferred lethality relies on persistence in the gut.

\section{Discussion}

Evf was initially identified as a virulence factor that promotes bacterial colonization of the Drosophila gut, yet also triggers a systemic immune response. A number of reports have documented the role of insects in general and Drosophilidae in particular in the dissemination of phytopathogenic bacteria such as Erwinia carotovora (Kloepper, 1981). The finding that evf was found only in a subset of Erwinia strains that have infectious properties towards Drosophila suggested that evf is an example of a gene that promotes survival and dissemination of bacteria in their environment. The goal of the present study was to analyse how Evf promotes colonization and persistence of bacteria inside the Drosophila larval gut. Our data show that Evf is not a toxin or a factor that antagonizes a specific host reaction of Drosophila. Rather, expression of this gene promotes the accumulation of bacteria in the anterior midgut dramatically affecting gut physiology. Interestingly, our study reveals unexpected reminiscences between the mechanisms of Evf mediated colonization of the Drosophila gut and the flea gut blockage induced by the plague agent Yersinia pestis (Hinnebusch et al., 2002).

In most cases, Gram-negative bacteria do not persist in the gut of Drosophila, indicating that the intestine is a rather hostile environment for invading bacteria (Ha et al., $2005 b)$. The mechanisms involved in bacterial clearance from the gut are poorly characterized, although recent studies have pointed towards a role of antimicrobial pep- tides and ROS in Drosophila adults. We have recently shown that the local expression of antimicrobial peptides in the gut, but not their systemic expression by the fat body, limits the growth of the entomopathogenic bacteria P. entomophila in Drosophila (Liehl et al., 2006). This illustrates the importance of Imd-mediated antibacterial responses in the gut against orally ingested bacteria. $P$. entomophila counteracts this response by secreting an zinc metalloprotease, AprA, which degrades antimicrobial peptides. A role for AprA in protection against the Imddependent immune response was supported by the observation that AprA was required to promote persistence in wt flies but was dispensable in a Relish background in which antimicrobial peptides active against Gram-negative bacteria are not produced. In contrast to the situation described above, we observed that evfdeficient bacteria did not persist in either wt or Relish mutant flies. This indicates that Evf does not provide any protection against antimicrobial peptides. This is corroborated by the observation that evf expressing bacteria did not resist against the Drosophila antimicrobial response when injected directly into the body cavity. Whereas a normal level of evf expression in Ecc15 does not induce larval lethality, overexpression on a multicopy plasmid (up to 50 -fold) induces lethality within $6 \mathrm{~h}$, a time frame too rapid for an efficient response involving antimicrobial peptides.

Natural gut infection has been associated with the rapid synthesis of ROS by the host, and the dynamics of ROS generation and elimination appears to be vital in Drosophila because flies that lack the capacity for ROS removal show an increased mortality after feeding with nonpathogenic bacteria (Ha et al., 2005b). Pathogenic bacteria are known to use specific responses to resist host ROS including the expression of detoxifying enzymes such as catalase (Harris et al., 2003). It was therefore tempting to predict a role for Evf in protection against ROS. However, in vitro assays failed to demonstrate a detoxifying activity against various chemicals including ROS or NO. The lethality induced upon infection by Ecc15 overexpressing evf was not suppressed in a fly line overexpressing a catalase (data not shown). It is interesting to note that Ecc15 bacteria do not appear to be more resistant to ROS species such as paraquat, hypochlorite or superoxide than naturally non-infectious bacteria, e.g. P. putida (C. Acosta Muniz, unpublished data).

Importantly, our observations that (i) evf mutants persisted in isolated guts equally well as wt Ecc15 and (ii) evf-expressing bacteria can exert an effect in trans on other bacteria do not support the hypothesis that Evf might be involved in the detoxification of compounds operative in gut bacterial clearance. Rather, persistence of evf mutants in isolated guts revealed that the elimination process requires the presence in situ of the gut in the 
A

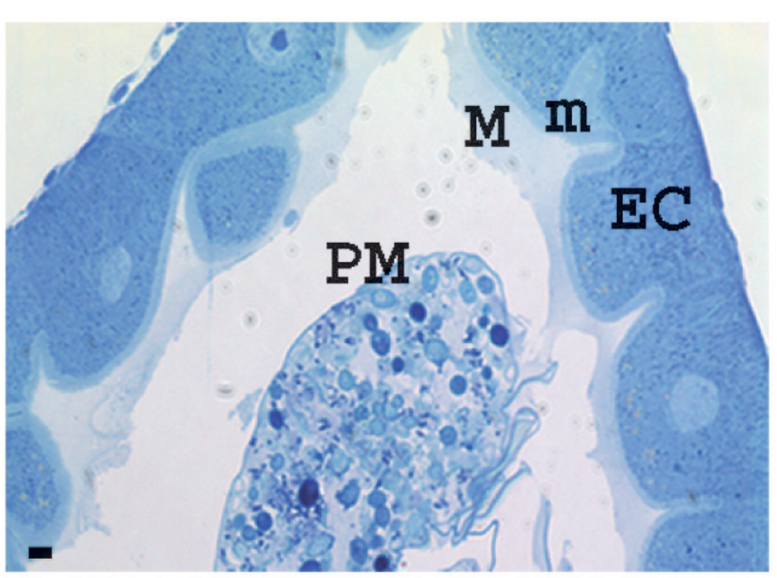

C

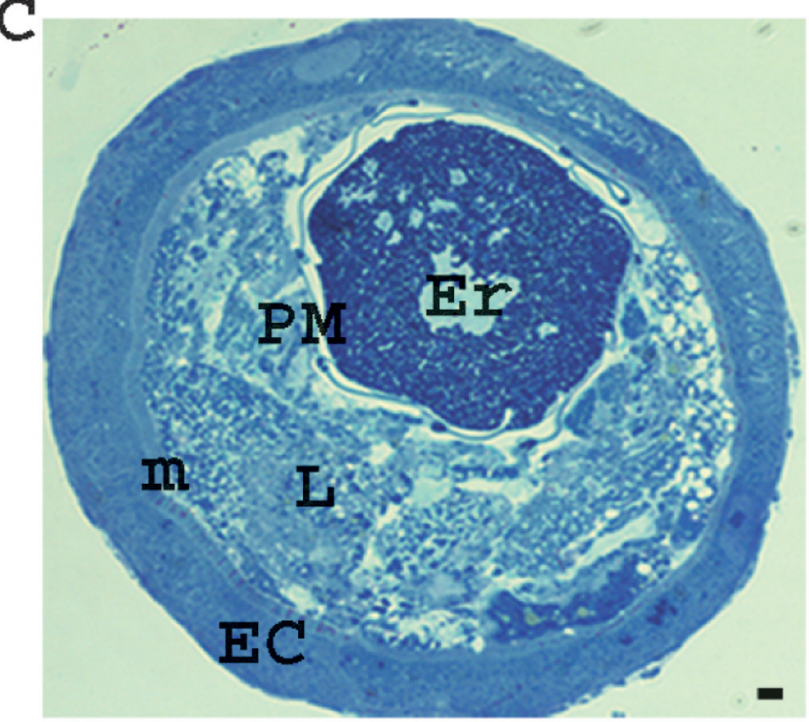

E

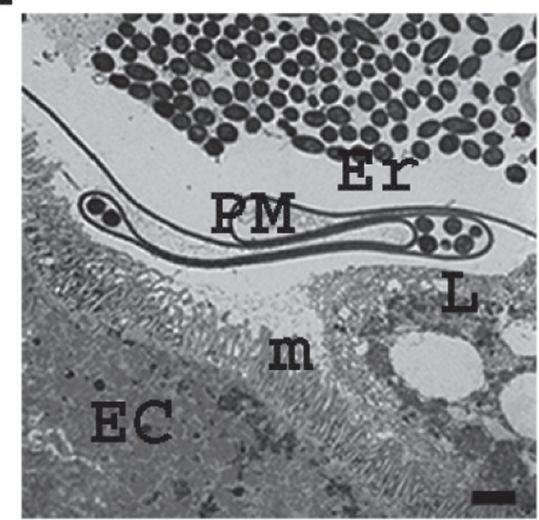

B

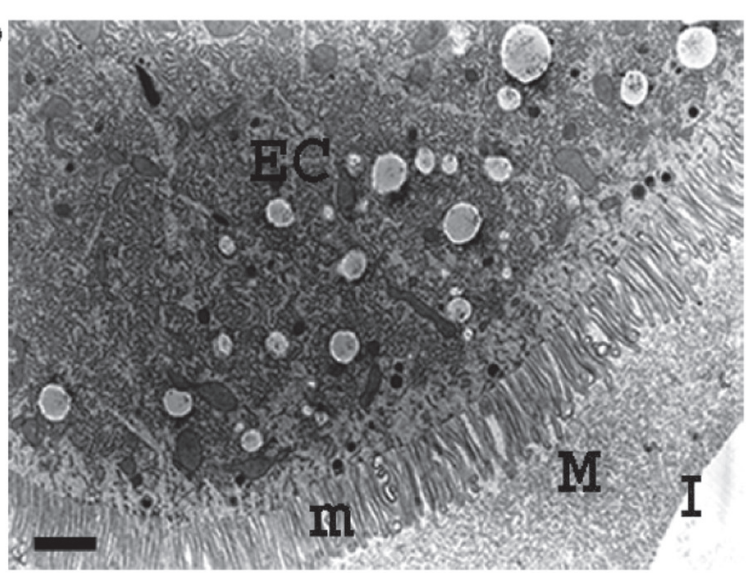

D

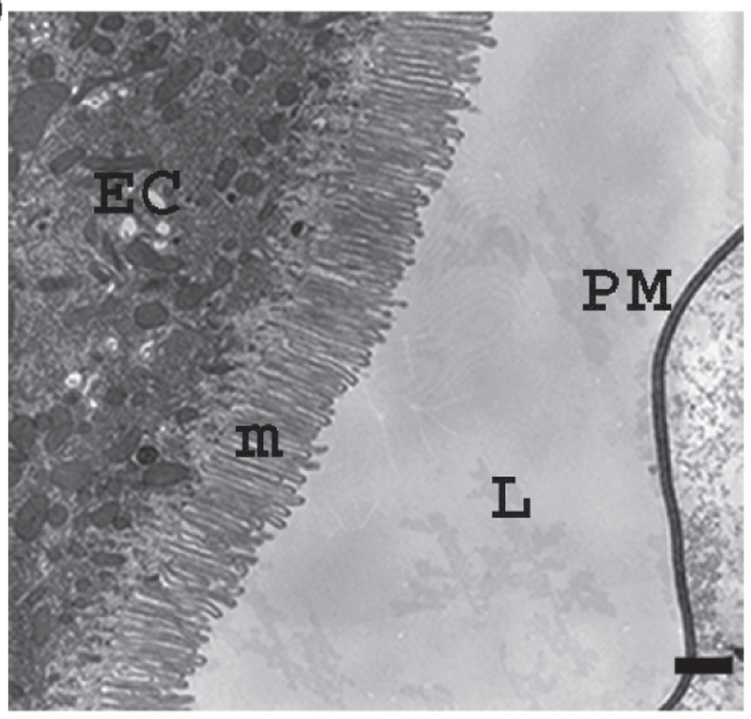

G

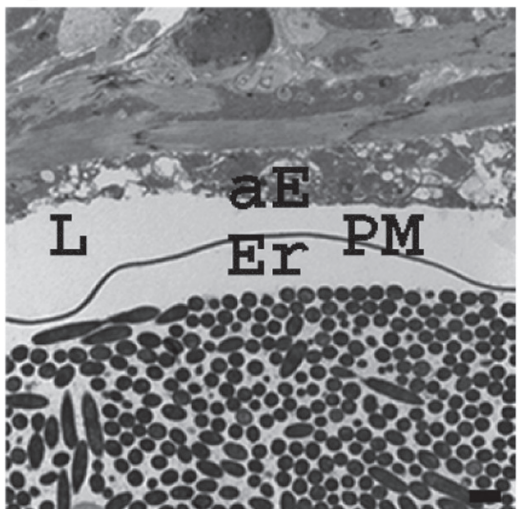

Fig. 6. evf overexpression in Ecc15 provokes a strong perturbation of the Drosophila larval gut. Transversal sections of larval anterior midguts collected at $2 \mathrm{~h} 30 \mathrm{~min}(\mathrm{~A}$ and $\mathrm{D})$ or $6 \mathrm{~h}(\mathrm{~B}-\mathrm{C}, \mathrm{E}-\mathrm{G})$ after natural infection with Ecc15 evf pOM1-evf $(\mathrm{C}-\mathrm{G})$ or control $(\mathrm{A}, \mathrm{B})$ were analysed. $(A, C)$ Semi-thin sections were observed under bright field. (B, D-G) Ultra-thin sections were observed by TEM. At $2 \mathrm{~h} 30 \mathrm{~min}$ and $6 \mathrm{~h}$ after infection, the mucus that protects the digestive epithelium was absent (compare B with $\mathrm{D}$ and $\mathrm{E}$ ). At $6 \mathrm{~h}$ after infection, the bacteria accumulated in the gut and the peritrophic matrix is not altered (C and $\mathrm{E}-\mathrm{G})$. At this time, the epithelial cells displayed abnormal microvilli and cellular material seemed to be present in the space between the epithelial cells and the peritrophic matrix (compare $\mathrm{D}$ with $\mathrm{E}$ and $\mathrm{F}$ ). $\mathrm{aE}$, epithelial cell absence; am, abnormal microvilli; EC, epithelial cell; Er, Ecc15 evf pOM1-evf; L, lumen; m, microvilli; M, mucus; PM, peritrophic matrix. (Scale bar; A, C and G, $10 \mu \mathrm{m}$; B- D, $3 \mu \mathrm{m} ; \mathrm{E}, \mathrm{F}$ and $\mathrm{H}, 2 \mu \mathrm{m}$ ). 
animal body. In addition, the effect of Evf is specific for gut persistence in larvae as no lethality was apparent after direct injection into the body cavity of bacteria overexpressing evf (our unpublished data). This indicates that Evf mediated persistence is specific to the physiology and architecture of the Drosophila larval gut.

To gather insight into the activity of Evf, we took advantage of the strong effect provoked by the 50 -fold overexpression of evf. This overexpression leads to a high level of bacterial accumulation inside the gut lumen. Although still being contained by the peritrophic matrix, a disappearance of mucus at the apical side of the epithelial cells and the appearance of cellular debris between epithelial cells and the peritrophic matrix can be observed. Ultimately, death of the larvae occur within 6-12 h following ingestion. Remarkably, microscopic analyses indicated that this large accumulation of bacteria does not break the peritrophic matrix and no direct contacts are apparent between bacterial and epithelial cells. The integrity of the peritrophic matrix despite the high bacterial load corroborates our previous observations that revealed spreading of bacteria across the gut barrier only in a minor fraction of larvae (Basset et al., 2003). We also noticed that the bacterial distribution was not random but rather seemed to follow a specific ordered arrangement that is reminiscent of an organized bacterial community such as a biofilm. Altogether, these results indicate that Evf activity may allow access of bacteria to a specific location, such as the proventriculus, by interfering locally with gut peristalsis. This colonization/proliferation hypothesis is reminiscent of the association of $Y$. pestis with its flea vector. Transmission of plague by fleas depends on infection of the proventriculus by a dense aggregate of $Y$. pestis cells organized in a biofilm that blocks normal blood feeding (Hinnebusch et al., 1996). From this point of view, it is striking that other entomopathogens such as $P$. entomophila and Serratia entomophila also induce an anti-feeding reaction that perturbs the physiology of the larval gut (Hurst et al., 2000; 2004; Vodovar et al., 2005). Together, these studies suggest that blockage of gut peristalsis, and thus food bolus movement, may be a common strategy used by entomopathogenic bacteria to circumvent elimination from the insect gut, although the molecular mechanisms may vary. Food movement through the gut is, of course, a necessary biological feature of the digestive process, yet may also be viewed as an important process for the elimination of potential pathogenic organisms. Microorganisms possessing a means to persist in the alimentary tract are thus at a natural advantage to avoid natural elimination.

In the absence of a direct functional homologue in another species or a tertiary structural protein signature, the nature of the molecular function of Evf still remains elusive. Our results indicate that Evf accumulates in the cytoplasm and its activity confers infectious properties only to Gram-negative bacteria. The ability of Evf to allow persistence in bacteria as diverse as E. coli or Pseudomonas species suggests that this protein plays a direct role in gut persistence of bacteria. It should be noted that only one similar open reading frame (ORF) can be identified in the databases upon a Psi BLAST search, which is plu2433 of the entomopathogen Photorhabdus luminescens TT01 (Duchaud et al., 2003). Although no evidence exists to date suggesting a role of PLU2433 in virulence, it is interesting that during the natural life-cycle of $P$. luminescens, the bacteria exist in a symbiotic relationship within the intestine of Heterorhabditis nematodes reinforcing the notion that these proteins may be involved in specific gut interactions.

In agreement with a direct role of Evf, the absence of any effects on the $E$. coli transcriptome and our inability to identify a suppressor gene should be noted. The localization of the protein in the cytoplasm as well as the low transcriptional level of evf detected in Ecc15 suggests that Evf does not function as a toxin. An attractive hypothesis is that Evf may interact with other proteins endogenous to many Gram-negative bacteria resulting in a modification of the bacterial cell structure that subsequently allows bacterial persistence and the formation of aggregates. This may promote the formation of a biofilm, as our microscopic data indicate, and/or the specific attachment to a receptor within the gut, such as chitin. The host range specificity of Evf for Gram-negative bacteria and the absence of a suppressor of evf indicate that Evf mediates its effect by itself or affect an essential cellular structure conserved among these types of bacteria such as components of the cell wall. Several colonization factors have been shown to be enzymes that modify the bacterial cell envelope. An example is the pagP locus of Bordetella bronchiseptica that encodes a palmitoyl transferase that modifies lipid $A$ as part of the adaptation of this organism required for persistent infection (Preston et al., 2003).

Infectious strategies of several pathogenic bacteria involve the manipulation of the host immune response. Many human pathogenic bacteria species trigger excessive inflammatory reactions that damage host tissues. A specific feature of bacteria expressing evf is the induction of both local and systemic host immune responses. The mechanisms that link Evf to immune activation are not yet fully understood. However, recent studies suggest that this effect may be a consequence of bacterial colonization rather than a direct effect of Evf per se. In support of this hypothesis, neither ingestion nor injection of pure Evf protein has an effect on host viability or the immune response (data not shown). We and others have also recently shown that peptidoglycan recognition proteins (PGRPs) with amidase activity degrade the peptidoglycan of Gram-negative bacteria and prevent the host immune 
Table 2. Bacterial strains and plasmids.

\begin{tabular}{|c|c|c|}
\hline Strains, plasmids & Description & Source or reference \\
\hline \multicolumn{3}{|l|}{ Bacterial strains } \\
\hline Erwinia carotovora 15 (Ecc15) & Wild type & Basset et al. (2003) \\
\hline Ecc15 evf & evf::Tn $10\left(\mathrm{Kan}^{\mathrm{R}}\right)$ & Basset et al. (2003) \\
\hline Escherichia coli K12 MG1655 & Wild type & Lab collection \\
\hline MG1656 & Wild type $\Delta$ lac Mlul & Espeli et al. (2001) \\
\hline $\mathrm{CC} 118$ & $\begin{array}{l}\text { araD1394(ara, leu)7697 } \triangle \text { lacX74 phoA-20 } \\
\quad \text { galE galK thi rpsE rpoB } \operatorname{argE}(A m) \text { recA1 }\end{array}$ & Manoil and Beckwith (1985) \\
\hline Pseudomonas aeruginosa PAO1 & Wild type & Gallagher et al. (2002) \\
\hline Pseudomonas putida KT2440 & Wild type & Laboratory collection \\
\hline Salmonella typhimurium LT2 & Wild type & Laboratory collection \\
\hline Bacillus subtilis 168 & Wild type & Laboratory collection \\
\hline Streptococcus faecalis JH2-2 & Wild type & Laboratory collection \\
\hline Bacillus megaterium & Wild type & Laboratory collection \\
\hline \multicolumn{3}{|l|}{ Plasmids } \\
\hline pOM1 & Cloning vector $\mathrm{pSC} 101$ derivative $\left(\mathrm{Spc}^{\mathrm{R}}\right)$ & Espeli et al. (2001) \\
\hline pOM3 & pOM1 expressing lacZ & Espeli et al. (2001) \\
\hline pOM1-GFP & pOM1 expressing gfp & Basset et al. (2003) \\
\hline pOM1-evf-GFP & pOM1 expressing evf and gfp & Basset et al. (2003) \\
\hline pX2-GFP & pX2 expressing gfp & Vodovar et al. (2005) \\
\hline pX2-evf-GFP & pX2 expressing gfp and evf & This study \\
\hline pDG148-GFP & pDG148 expressing gfp & Joseph et al. (2001) \\
\hline pDG148-evf-GFP & pDG148 expressing gfp and evf & This study \\
\hline pMC1403 & ColE1, lacZ gene fusion vector & Casadaban et al. (1980) \\
\hline pPHO7 & phoA gene fusion vector & Gutierrez and Devedjian (1989) \\
\hline pNKBOR & R6K derivative carying a mini-Tn 10 -based transposon & Rossignol et al. (2001) \\
\hline pHK11-Amp & HK022-based integrative vector & Rossignol et al. (2002) \\
\hline pHK-int & pSC101 derivative expressing integrase & Rossignol et al. (2002) \\
\hline pHK11-pR-evf-lacZ & pHK11-Amp carrying $P_{R}$-evf-lacZ & This study \\
\hline
\end{tabular}

response of flies to the presence of ingested bacteria in the gut (Bischoff et al., 2006; Zaidman-Rémy et al., 2006). This activity might be a natural host mechanism allowing the establishment of a tolerance threshold level of bacteria in the gut, presumably present in natural ingested food, thereby avoiding over-stimulation of the immune response under normal conditions. Moreover, it was also proposed that the systemic immune response induced by persistent and infectious bacteria such as Ecc15 is mediated by the translocation of small peptidoglycan fragments from the gut lumen to the haemolymph. Our data are compatible with a model in which bacterial persistence in the gut leads to a local increase of the peptidoglycan concentration that exceeds the host tolerance level and results in stimulation of the immune system. This is consistent with our observation that Evf does not promote crossing of the gut barrier by bacteria. As peptidoglycan is expected to be able to cross the peritrophic matrix, it remains intriguing that high titres of ingested Gramnegative bacteria like $E$. coli do not induce the immune response although high numbers of bacteria are still present in the gut several hours after ingestion. The paradoxical absence of immune response activation despite the presence of high numbers of cells at early time points after ingestion indicates that triggering of the immune response by infectious bacteria such as Ecc15 either requires bacterial proliferation and detection of de novo synthesized peptidoglycan compounds or alternatively depends on detection of peptidoglycan molecules in a specific compartment of the gut.

Further work will be necessary to identify the exact biological activity of Evf and to determine whether gut tissues in Drosophila constitute the site of initial bacterial colonization and which are the cell types involved in bacterial recognition.

\section{Experimental procedures}

Drosophila stocks

Oregon $^{R}\left(\right.$ Or $\left.^{R}\right)$ flies were used as a standard wt strain. Relish ${ }^{E 20}$ flies carry a null mutation in Relish that encodes the transactivator regulated by the Imd pathway (Hedengren et al., 1999). Drosophila stocks were maintained at $25^{\circ} \mathrm{C}$.

\section{Bacterial strains}

The strains used in this study are listed in Table 2. Bacteria were cultured in Lennox medium with the appropriate antibiotics (100 $\mu \mathrm{g} \mathrm{ml}^{-1}$ rifampicin; $100 \mu \mathrm{g} \mathrm{ml}^{-1}$ ampicillin; $300 \mu \mathrm{g} \mathrm{ml}^{-1}$ carbenicillin; kanamycin $50 \mu \mathrm{g} \mathrm{ml}^{-1}$ and $100 \mu \mathrm{g} \mathrm{ml}^{-1}$ spectinomycin). The rifampicin-resistant Ecc15, Ecc15 derivatives and Pseudomonas were grown at $29^{\circ} \mathrm{C}$. E. coli strains were grown at $37^{\circ} \mathrm{C}$ if not otherwise indicated.

\section{Chemicals}

Analytical grade $\mathrm{H}_{2} \mathrm{O}_{2}$, paraquat and $\mathrm{NaClO}$, were purchased from Sigma. Diethylenetriamine (DETA) NONOate ( \pm )-S-Nitroso- 
$\mathrm{N}$-acetylpenicillamine (SNAP), S-Nitrosoglutathione (GSNO), SIN-1 Hydrochloride and Spermine NONOate were a kind gift from Jean-Claude Drapier (Institut de Chimie des Substances Naturelles, CNRS, Gif-sur-Yvette) and purchased from Calbiochem.

\section{Drosophila natural bacterial infection}

Approximately 200 third-instar larvae were placed in a $2 \mathrm{ml}$ tube containing $200 \mu \mathrm{l}$ of concentrated bacteria pellet $\left(\mathrm{OD}_{600}=200\right)$ from an overnight culture and $400 \mu \mathrm{l}$ of crushed banana. The larva, bacteria and banana were thoroughly mixed in the microfuge tube; the tube was closed with a foam plug, incubated at room temperature for $30 \mathrm{~min}$, and the mix was then transferred to a standard corn-meal fly medium and incubated at $29^{\circ} \mathrm{C}$. For bacterial counting experiments, the infected larvae were first rinsed in water and transferred to a fresh fly medium at $2 \mathrm{~h}$ after infection. Counting procedures were performed with larvae rinsed in water and dipped in $70 \%$ ethanol (three times for $5 \mathrm{~s}$ ) for external sterilization and then homogenized and spread onto Luria-Bertani (LB) plates containing the required antibiotic at each different time point. For bacterial counting in isolated guts, larvae were dissected $2 \mathrm{~h}$ after infection in Drosophila Schneider medium after ethanol sterilization, and the guts were placed in Schneider medium with $10 \%$ bovine serum, homogenized and spread onto LB plates containing antibiotics at each time point. Bacterial injections of adults were performed by pricking adults in the thorax with a thin needle previously dipped into a concentrated pellet of a bacterial culture $\left(\mathrm{OD}_{600}=200\right)$.

\section{Transmission electron microscopy (TEM)}

Infected Drosophila larvae were dissected in Schneider medium, and the guts were immediately fixed with $2.5 \%$ glutaraldehyde, $1 \%$ paraformaldehyde, $1 \%$ potassium ferrocianide solution in $0.1 \mathrm{M}$ cacodylate buffer, $\mathrm{pH} 7.4$, for $80 \mathrm{~min}$ at room temperature. Dehydration of the guts was performed in an ascending series of ethanol concentrations, and then the samples were embedded in Epon 812. The guts were cut at $0.5 \mu \mathrm{m}$ semi-thin sections for light microscopy or $60 \mathrm{~nm}$ ultra-thin sections for TEM with a Leica Ultramicrotome. Semi-thin sections were stained with methylene blue and Azur II and observed under an Axiophot Zeiss microscope. Ultra-thin sections were contrasted with uranyl acetate and lead citrate and observed with a Philips 208 electron microscope.

\section{Microscopic observations of larvae}

Live larvae infected with GFP expressing bacteria were anesthetized on ice and viewed under epifluorescent illumination (excitation filter $480 / 40 \mathrm{~nm}$; dichroic filter $505 \mathrm{~nm}$; emission filter $510 \mathrm{~nm}$ ) with a Leica (Heerburg, Switzerland) MZFLIII dissecting microscope. Images were recorded with a charge-coupled device camera (Nikon).

\section{Midgut organs cultures}

Midguts were isolated from infected Drosophila and incubated in Schneider Drosophila Medium (Gibco) supplemented with 10\% fetal bovine serum (Biomedia).

\section{DNA manipulations}

All DNA manipulations, restriction digestions, ligations and transformations were performed using standard genetic and molecular techniques (Sambrook et al., 1989; Miller, 1992). Plasmid DNA was purified using a Quiagen kit. Restriction and DNA modifying enzymes were obtained from Boehringer Mannheim or New England Biolabs and used according to the manufacturers' instructions. PCR reactions were performed in a $50 \mu \mathrm{lmix}$ for 30 cycles using Phusion High-fidelity DNA polymerase (Finnzymes) according to the manufacturer's instructions in a DNA thermal cycler PTC-100 (MJ Research). PCR products were purified with the QIAquik kit (Qiagen) before and after digestion of the amplification product.

Plasmids used in this study are listed in Table 2. $\mathrm{pF} 1$ and pF2 were constructed by inserting PCR fragments harbouring different portions of the evf promoter region into pOM3 (Espeli et al., 2001) cut with EcoRI and Sall. Plasmid pF3 was constructed by deletion of the EcoRI-Sall fragment of pOM3 (Fig. 2A). The PCR products were generated using the downstream primer 5'-ATGCTAGTCGACAATCACTCCTATT GTGGTGG-3' and the upstream primers $5^{\prime}$-ATGCTAGAATT CATTTACTCACGAAAAATT-3' (pF1) and 5'-ATCGATGAATT CTATCTTTAATTATGGTTA-3' (pF2) and cut with Sall and EcoRI.

\section{Constructions of evf-lacZ and evf-phoA gene fusions}

L1-L3 fusions (Fig. 3) were constructed by inserting different regions of evf gene amplified by PCR using the plasmid pOM1evf (Basset et al., 2003) into pMC1403 (Casadaban et al., 1980) cut with EcoRI. The PCR products were generated using the upstream primer 5'-GGAATCTAGACATTCAGTTCGCTGC-3' and the downstream primers $5^{\prime}$-ATCGACGAATTCCTTTGG CTACTTCAACGCCTTTTAC-3' (L1), 5'-ATCGACGAATTCCGG TTTTATTCATTTCGGCACTTAAACC-3' (L2) and 5'-ATCGAC GAATTCCATATACATAATTTTTATTTGG-3' (L3) and cut with EcoRI (located in the upstream region of evf and in the downstream primers). P1-P3 fusions were constructed by inserting PCR fragments harbouring different portions of evf gene into pPHO7 (Gutierrez and Devedjian, 1989) cut by Hindlll and using the plasmid pOM1-evf like template. The PCR products were generated using the upstream primer 5'-ATCGACAAG CTTGAATTCGAGCTCGGTACCCCC- $3^{\prime}$ and the downstream primers 5'-ATCGACAAGCTTTTGGCTACTTCAACGCCTTT-3' (P1), 5'-ATCGACAAGCTTTTATTCATTTCGGCACTTAA-3' (P2) and 5'-ATCGACAAGCTTACATAATTTTTATTTGGCTT-3' (P3). P4 fusion was constructed by inserting a PCR fragment of ma/E gene, that contains the signal peptide for the exportation to the periplasm, in pPHO7 cut by HindlII. The PCR product was generated using the same upstream primer that in the other phoA fusions and the downstream primer $5^{\prime}$-ATCGACAAGCTTTT AGTCTGCGCGTCTTTCAGGGC-3'. The PCR products were digested with HindIII.

\section{Integration of the $P_{R}$-evf-lacZ operon in the $\mathrm{E}$. coli chromosome}

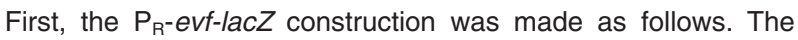
plasmid pOM1-evf was digested by EcoRI and HindIII, the $1 \mathrm{~kb}$ 
fragment was ligated in the pHK11-Amp (Rossignol et al., 2002) digested with EcoRI and HindIII. The $\mathrm{P}_{\mathrm{R}}$ promoter was cloned as a double strand oligonucleotide in the pHK11-evf digested with EcoRI and Pstl. The lac $Z$ gene was obtained from pOM3 digested with Sall, filled in with Klenow fragment and digested with HindIII. lacZ was subsequently cloned in the pHK11- $\mathrm{P}_{\mathrm{R}}$-evf digested with $\mathrm{Ncol}$, filled in by Klenow fragment and digested with Hindlll. For integration of $\mathrm{P}_{\mathrm{R}}$-evf-lacZ operon in the E. coli chromosome, we used the system which allows the insertion of DNA by site-specific integration into the bacteriophage HK022 bacterial attachment site (Rossignol et al., 2002).

\section{Random mutagenesis of the 'E. coli pR-evf-lacZ'}

For the construction of mutants we used a mini-Tn10 based transposon NKBOR as described previously (Rossignol et al., 2001).

\section{Transcriptome analysis of E. coli strains carrying pOM1 and pOM1-evf}

Total RNAs of strains containing pOM1 and pOM1-evf were extracted from exponential cultures as described before (Espeli et al., 2001). cDNAs produced from total RNA isolated from E. coli MG1655 $\Delta$ laclZ carrying pOM1 or pOM1-evf strains were Cy-3 and Cy-5 labelled respectively, and hybridized on E. coli DNA chips carrying the complete set of $E$. coli ORFs. DNA chips were prepared in the Gif/Orsay DNA Microarray Platform (GODMAP).

\section{$\beta$-Galactosidase and alkaline phosphatase assays}

$\beta$-Galactosidase and alkaline phosphatase activities were measured in permeabilized cells as described previously (Miller, 1992; Manoil, 2000). $\beta$-Galactosidase and alkaline phosphatase assays were performed in triplicate.

\section{Acknowledgements}

We thank I. Attree, S. Gruss, C. Manoil, Ph. Bouloc, P. Serror, J.-L. Drapier for providing bacterial strains and reagents, A. Jacq for helpful discussions, Christoph Scherfer, Peter Liehl and Sean Kennedy for critical reading of the manuscript and Brigitte Maroni and Michèle Valens for technical help. C.A. was supported by a doctoral fellowship from CONACYT (Mexico). The laboratories of F. B and B. L. were funded, respectively, by CNRS and by CNRS, the Agence Nationale de la Recherche, the Schlumberger and Bettencourt Foundations and the ACI Microbiologie.

\section{References}

Basset, A., Khush, R.S., Braun, A., Gardan, L., Boccard, F., Hoffmann, J.A., and Lemaitre, B. (2000) The phytopathogenic bacteria Erwinia carotovora infects Drosophila and activates an immune response. Proc Natl Acad Sci USA 97: 3376-3381.

Basset, A., Tzou, P., Lemaitre, B., and Boccard, F. (2003) A single gene that promotes interaction of a phytopathogenic bacterium with its insect vector, Drosophila melanogaster. EMBO Rep 4: 205-209.

Bischoff, V., Vignal, C., Duvic, B., Boneca, I.G., Hoffman, J.A., and Royet, J. (2006) Downregulation of the Drosophila immune response by peptidoglycan-recognition proteins SC1 and SC2. PLoS Pathog 2: e14.

Casadaban, M.J., Chou, J., and Cohen, S.N. (1980) In vitro gene fusions that join an enzymatically active betagalactosidase segment to amino-terminal fragments of exogenous proteins: Escherichia coli plasmid vectors for the detection and cloning of translational initiation signals. $J$ Bacteriol 143: 971-980.

Chugani, S.A., Whiteley, M., Lee, K.M., D’Argenio, D., Manoil, C., and Greenberg, E.P. (2001) QscR, a modulator of quorum-sensing signal synthesis and virulence in Pseudomonas aeruginosa. Proc Natl Acad Sci USA 98: 2752-2757.

Deng, S., Stein, R.A., and Higgins, N.P. (2004) Transcriptioninduced barriers to supercoil diffusion in the Salmonella typhimurium chromosome. Proc Natl Acad Sci USA 101: 3398-3403. Epub 2004 March 3391.

Duchaud, E., Rusniok, C., Frangeul, L., Buchrieser, C., Givaudan, A., Taourit, S., et al. (2003) The genome sequence of the entomopathogenic bacterium Photorhabdus luminescens. Nat Biotechnol 21: 1307-1313. Epub 2003 October 1305.

Espeli, O., Moulin, L., and Boccard, F. (2001) Transcription attenuation associated with bacterial repetitive extragenic BIME elements. J Mol Biol 314: 375-386.

Flyg, C., Kenne, K., and Boman, H.G. (1980) Insect pathogenic properties of Serratia marcescens: phage-resistant mutants with a decreased resistance to Cecropia immunity and a decreased virulence to Drosophila. J Gen Microbiol 120: 173-181.

Gallagher, L.A., McKnight, S.L., Kuznetsova, M.S., Pesci, E.C., and Manoil, C. (2002) Functions required for extracellular quinolone signaling by Pseudomonas aeruginosa. J Bacteriol 184: 6472-6480.

Gutierrez, C., and Devedjian, J.C. (1989) A plasmid facilitating in vitro construction of phoA gene fusions in Escherichia coli. Nucleic Acids Res 17: 3999.

Ha, E.M., Oh, C.T., Bae, Y.S., and Lee, W.J. (2005a) A direct role for dual oxidase in Drosophila gut immunity. Science 310: 847-850.

Ha, E.M., Oh, C.T., Ryu, J.H., Bae, Y.S., Kang, S.W., Jang, I.H., et al. (2005b) An antioxidant system required for host protection against gut infection in Drosophila. Dev Cell 8: 125-132.

Harris, A.G., Wilson, J.E., Danon, S.J., Dixon, M.F., Donegan, K., and Hazell, S.L. (2003) Catalase (KatA) and KatA-associated protein (KapA) are essential to persistent colonization in the Helicobacter pylori SS1 mouse model. Microbiology 149: 665-672.

Hedengren, M., Asling, B., Dushay, M.S., Ando, I., Ekengren, S., Wihlborg, M., and Hultmark, D. (1999) Relish, a central factor in the control of humoral but not cellular immunity in Drosophila. Mol Cell 4: 827-837.

Hinnebusch, B.J., Perry, R.D., and Schwan, T.G. (1996) Role of the Yersinia pestis hemin storage (hms) locus in the transmission of plague by fleas. Science 273: 367370. 
Hinnebusch, B.J., Rudolph, A.E., Cherepanov, P., Dixon, J.E., Schwan, T.G., and Forsberg, A. (2002) Role of Yersinia murine toxin in survival of Yersinia pestis in the midgut of the flea vector. Science 296: 733-735.

Hultmark, D. (2003) Drosophila immunity: paths and patterns. Curr Opin Immunol 15: 12-19.

Hurst, M.R., Glare, T.R., Jackson, T.A., and Ronson, C.W. (2000) Plasmid-located pathogenicity determinants of Serratia entomophila, the causal agent of amber disease of grass grub, show similarity to the insecticidal toxins of Photorhabdus luminescens. J Bacteriol 182: 5127-5138.

Hurst, M.R., Glare, T.R., and Jackson, T.A. (2004) Cloning Serratia entomophila antifeeding genes - a putative defective prophage active against the grass grub Costelytra zealandica. J Bacteriol 186: 5116-5128.

Joseph, P., Fantino, J.R., Herbaud, M.L., and Denizot, F. (2001) Rapid orientated cloning in a shuttle vector allowing modulated gene expression in Bacillus subtilis. FEMS Microbiol Lett 205: 91-97.

Kloepper, J.W., Brewer, J.W., and Harrison, M.D. (1981) Insect transmission of Erwinia carotovora var. carotovora and Erwinia carotovora var. atroseptica to potato plants in the field. Am Potato J 58: 165-175.

Liehl, P., Blight, M., Vodovar, N., Boccard, F., and Lemaitre, B. (2006) Prevalence of local immune response against oral infection in a Drosophila/Pseudomonas infection model. PLOS pathogen 2: e56.

de Maagd, R.A., Bravo, A., and Crickmore, N. (2001) How Bacillus thuringiensis has evolved specific toxins to colonize the insect world. Trends Genet 17: 193-199.

Manoil, C. (2000) Tagging exported proteins using Escherichia coli alkaline phosphatase gene fusions. Methods Enzymol 326: 35-47.

Manoil, C., and Beckwith, J. (1985) TnphoA: a transponson probe for protein export signals. Proc Natl Acad Sci U S A 82: 8129-8133.
Miller, J. (1992) Experiments in Molecular Genetics. Cold Spring Harbor, NY: Cold Spring Harbor Laboratory Press.

Preston, A., Maxim, E., Toland, E., Pishko, E.J., Harvill, E.T., Caroff, M., and Maskell, D.J. (2003) Bordetella bronchiseptica PagP is a Bvg-regulated lipid A palmitoyl transferase that is required for persistent colonization of the mouse respiratory tract. Mol Microbiol 48: 725-736.

Rossignol, M., Basset, A., Espeli, O., and Boccard, F. (2001) NKBOR, a mini-Tn10-based transposon for random insertion in the chromosome of Gram-negative bacteria and the rapid recovery of sequences flanking the insertion sites in Escherichia coli. Res Microbiol 152: 481-485.

Rossignol, M., Moulin, L., and Boccard, F. (2002) Phage HK022-based integrative vectors for the insertion of genes in the chromosome of multiply marked Escherichia coli strains. FEMS Microbiol Lett 213: 45-49.

Sambrook, J., Fritsch, E.F., and Maniatis, T. (1989) Molecular Cloning: A Laboratory Manual. Cold Spring Harbor, NY: Cold Spring Harbor Laboratory Press.

Thomson, N.R., Cox, A., Bycroft, B.W., Stewart, G.S., Williams, P., and Salmond, G.P. (1997) The rap and hor proteins of Erwinia, Serratia and Yersinia: a novel subgroup in a growing superfamily of proteins regulating diverse physiological processes in bacterial pathogens. Mol Microbiol 26: $531-544$.

Vodovar, N., Acosta, C., Lemaitre, B., and Boccard, F. (2004) Drosophila: a polyvalent model to decipher host-pathogen interactions. Trends Microbiol 12: 235-242.

Vodovar, N., Vinals, M., Liehl, P., Basset, A., Degrouard, J., Spellman, P., et al. (2005) Drosophila host defense after oral infection by an entomopathogenic Pseudomonas species. Proc Natl Acad Sci USA 102: 11414-11419.

Zaidman-Rémy, A., Hervé, M., Poidevin, M., Pili-Floury, S., Kim, M.-S., Blanot, D., et al. (2006) The Drosophila amidase PGRP-LB modulates the immune response to bacterial infection. Immunity 24: 463-473. 